\title{
COMPOSITE FLEXURAL MODE RESONATOR WITH REDUCED TEMPERATURE COEFFICIENT OF FREQUENCY
}

\author{
R. Melamud, B. Kim, M. A. Hopcroft, S. Chandorkar, M. Agarwal, \\ C.Jha, S. Bhat, K. K. Park, and T. W. Kenny \\ Departments of Mechanical and Electrical Engineering, Stanford University \\ Stanford, CA, USA
}

\begin{abstract}
This paper reports a method to passively reduce the native temperature coefficient of frequency (TCF) by creating a Silicon - Silicon Dioxide $\left(\mathrm{Si}_{-} \mathrm{SiO}_{2}\right)$ composite resonator whose bending stiffness has reduced sensitivity to temperature. Silicon exhibits softening with increasing temperature. Amorphous silicon dioxide exhibits an anomalous hardening with increasing temperature. An optimal combination of $\mathrm{Si}_{-} \mathrm{SiO}_{2}$ in flexural mode resonators is determined that would yield zero TCF. Composite single anchor, double ended tuning forks (DETF) where fabricated and show a greater than $10 \mathrm{x}$ reduction in TCF in the $15^{\circ} \mathrm{C}$ to $70^{\circ} \mathrm{C}$ temperature range. The composite beam resonators were fabricated in our wafer-scale encapsulation process, which is described by [1] and is being commercialized.
\end{abstract}

\section{INTRODUCTION}

To become a viable replacement for the ubiquitous quartz crystal technology, silicon resonators must have a frequency stability of under $100 \mathrm{ppm}$ in the $0^{\circ} \mathrm{C}$ to $70^{\circ} \mathrm{C}$ temperature range. Silicon resonators exhibit a native temperature coefficient of frequency (TCF) of $-30 \mathrm{ppm} /{ }^{\circ} \mathrm{C}$ that is dominated by the decrease of the resonator stiffness due to the material softening of Silicon.

Current compensation methods can be classified as either active or passive. Active compensation techniques such as ovenization [2] and electrostatic tuning [3] require power in order to reduce the TCF into the desirable range. Passive compensation methods attempt to reduce the native TCF without application of power. Previous passive compensation techniques [4,5] have used axial stress induction in flexural mode resonators to shift the natural frequency. This was accomplished by using materials with different coefficients of thermal expansion (CTE) that would induce thermal stresses with changes in temperature. Although these methods demonstrated the ability to reduce TCF, introducing materials with mismatched CTE's can cause stress induced hysteresis, processing difficulties due to large thermal stresses, restrictions on material selection for clean processes, and undesirable sensitivity to package stress.

This paper reports a method to passively reduce the native TCF by creating a Silicon - Silicon Dioxide composite resonator whose bending stiffness can be insensitive to temperature variation. Silicon dioxide exhibits an anomalous temperature coefficient of Young's Modulus (TCE) that is positive [6]. By combining it with Silicon, which has a negative TCE, it is possible to reduce the temperature dependence of the stiffness of the resonator. Furthermore, this method does not use stress induction as the means to compensate frequency.

\section{THEORY}

Flexural mode beam-type resonators, such as the double end tuning forks used in this paper (Fig. 1), that are fabricated from a single material and are single anchored have a frequency and

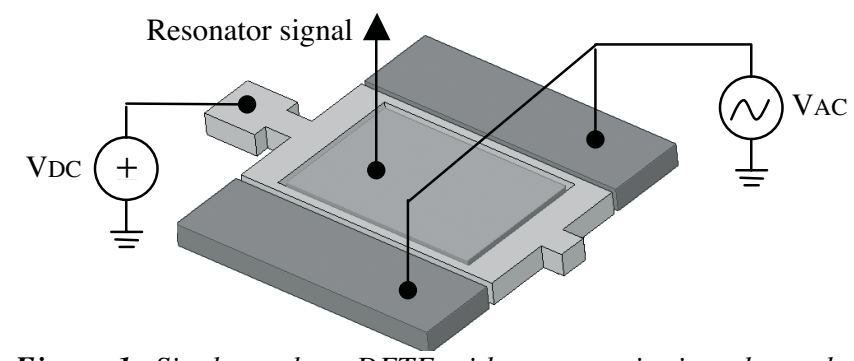

Figure 1. Single anchor, DETF with outer excitation electrodes and an inner sense electrode.

TCF described in Equation 1. Equation 1.b ignores the smaller effect of dimensional changes with temperature and assumes linear material properties.

$$
\begin{aligned}
& f=\frac{\beta^{2}}{2 \pi L^{2}} \sqrt{\frac{B}{\rho A}}=\frac{\beta^{2}}{2 \pi L^{2}} \sqrt{\frac{E I}{\rho A}} \\
& T C F=\frac{1}{f_{o}} \frac{\partial f}{\partial T} \approx \frac{1}{2} \frac{\partial f}{\partial B} \frac{\partial B}{\partial T} \approx \frac{T C E}{2}
\end{aligned}
$$

In the above equations, $\beta$ is the mode constant, $E$ is the Young's Modulus, $\rho$ is the density of the material, $I$ is the flexural rigidity, $L$ is the length of the beam, $A$ is the cross sectional area of the beam, $B=E I$ is defined as the bending stiffness of the beam, $f_{\mathrm{o}}$ is a reference frequency, and TCE is the temperature coefficient of Young's modulus of the beam's material. The TCE of Silicon is approximately $-60 \mathrm{ppm} /{ }^{\circ} \mathrm{C}$ which results in a TCF of $-30 \mathrm{ppm} /{ }^{\circ} \mathrm{C}$ for Silicon resonators.

For a composite beam of $\mathrm{N}$ materials, the above equations are modified such that the bending stiffness takes into account the geometric composition of the beam (Eq. 2a).

$$
\begin{aligned}
& B=\sum_{j=1}^{N} E_{j} I_{j} \\
& T C F \approx \frac{1}{2}\left[\sum_{j=1}^{N} E_{j} I_{j} T C E_{j} / \sum_{j=1}^{N} E_{j} I_{j}\right]
\end{aligned}
$$

The resulting modified TCF is a function of the TCE of each of the materials in the composite. By using amorphous $\mathrm{SiO}_{2}$, which has a positive temperature coefficient of Young's Modulus (TCE) of $+195 \mathrm{ppm} /{ }^{\circ} \mathrm{C}$, in combination with Silicon, it is possible to design an ideal composite beam whose bending stiffness does not change with temperature yielding a zero TCF resonator.

From the above equation, the only design parameter that can be controlled is the flexural rigidity of each material. For a Si$\mathrm{SiO}_{2}$ composite, the optimal configuration requires the minimal amount of $\mathrm{SiO}_{2}$ growth in order to achieve zero TCF. Several configurations have been considered (Fig 2). A laminate type structure (Fig 2.a) has a flexural rigidity that is linearly related to the thickness of the $\mathrm{SiO}_{2}$. However, an encapsulated structure (Fig 2.b) has a quartic dependence on thickness of the $\mathrm{SiO}_{2}$. The latter configuration was chosen since it is most sensitive to $\mathrm{SiO}_{2}$ thickness and can be easily achieved by oxidizing all exposed Silicon surfaces. Figure 3 shows the result of evaluating the composite beam TCF equation (Eq. 2.b) for the optimal 
configuration (Fig 2.b). The target thickness necessary to achieve zero TCF depends on the width of the beam. The model also took into account the orientation dependent oxidation of the beam surfaces as well as the consumption of Silicon during oxidation $\left(0.46 \mu \mathrm{m}\right.$ of $\mathrm{Si}$ for $1 \mu \mathrm{m}$ of $\left.\mathrm{SiO}_{2}\right)$ [7].

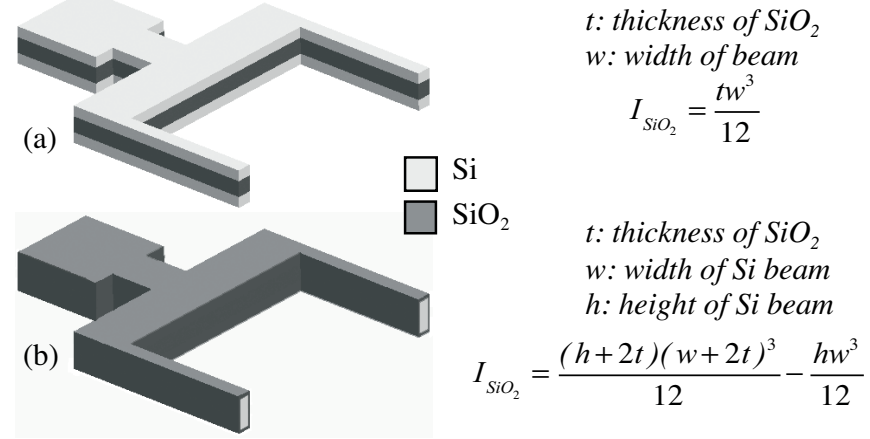

Figure 2. Cross-section of the DETF through the beams of the resonator. The TCF of the composites will depend on the thickness of the $\mathrm{SiO}_{2}$ layers. (a) Laminate construction with $\mathrm{SiO}_{2}$ sandwiched between Si layers. (b) $\mathrm{SiO}_{2}$ coated $\mathrm{Si}$ by oxidation of all $\mathrm{Si}$ surfaces. The oxidized configuration is optimal since the flexural rigidity, I, of the $\mathrm{SiO}_{2}$ is most sensitive to the thickness of $\mathrm{SiO}_{2}$

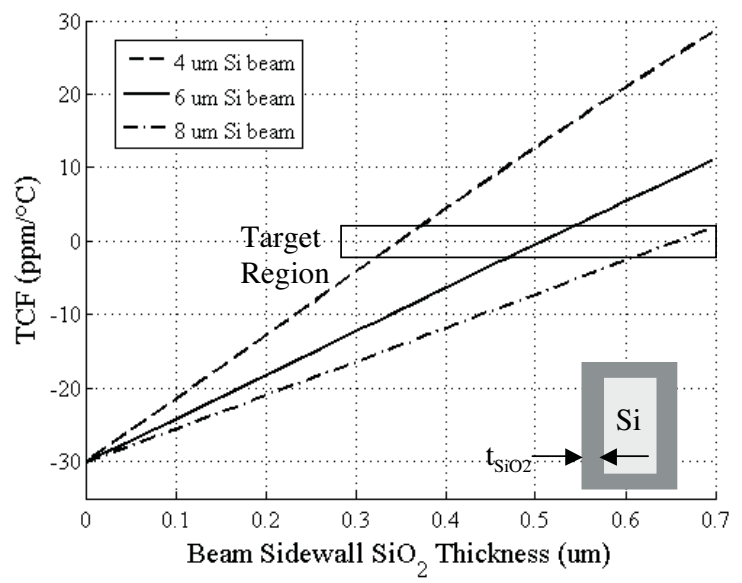

Figure 3. $\mathrm{SiO}_{2}$ thickness on sidewall of beam versus TCF for oxidized silicon resonator configuration (Fig 2.b). This model takes into account orientation dependent oxidation rates and consumption of silicon during oxidation. The target oxidation depends on the initial width of the Si beam. The constants used in this model are: $T C E_{S i}=-60 \mathrm{ppm} /{ }^{\circ} \mathrm{C}, \quad \mathrm{TCE}_{\mathrm{SiO} 2}=+195 \mathrm{ppm} /{ }^{\circ} \mathrm{C}$, $E_{\mathrm{Si}}=168 \mathrm{GPa}, E_{\mathrm{SiO} 2}=70 \mathrm{GPa}, h=20 \mu \mathrm{m}$.

\section{FABRICATION AND EXPERIMENTS}

$\mathrm{Si}-\mathrm{SiO}_{2}$ composite resonators were fabricated using a wafer scale polysilicon encapsulation with oxide seal processes described in [1]. The only modification was a 30 minute wet oxidation at $1100^{\circ} \mathrm{C}$ to oxidize the surfaces of the resonator beams. The oxidation occurred after the resonator was released by HF vapor etching of the sacrificial oxide. The thickness of the oxidation was approximated by measuring the $\mathrm{SiO}_{2}$ thickness $(0.44$ $\mu \mathrm{m})$ on the surface of the encapsulation. The oxidized resonators, with $7.5 \mu \mathrm{m}$ beam widths, were measured at $40^{\circ} \mathrm{C}$ and over a $15^{\circ} \mathrm{C}$ to $70^{\circ} \mathrm{C}$ temperature range using a temperature controlled oven.

The oxide seal process used for these preliminary experiments in not as clean as our high temperature epi-polysilicon seal. Devices sealed in this process were operated at a fixed $40^{\circ} \mathrm{C}$ temperature for 3 hours and showed a positive frequency drift of $\sim 11 \mathrm{ppm} / \mathrm{hr}$. This drift is attributed to migration of absorbents off of the resonators. The drift is slow enough to allow our first experiments on the temperature dependence of frequency for oxide/silicon composite beams.

Frequency versus temperature data was taken for five cycles over a temperature variation from $15^{\circ} \mathrm{C}$ to $70^{\circ} \mathrm{C}$. The experiment took place over a continuous $15 \mathrm{hr}$ time span and a $+9.9 \mathrm{ppm} / \mathrm{hr}$ drift was observed. The total frequency excursion during this time span was $260 \mathrm{ppm}$. However, approximately 150 $\mathrm{ppm}$ is attributed to a linear frequency drift over time. Figure 4 shows the frequency versus temperature data for the five cycles as well as a comparison to the native TCF of a silicon DETF.

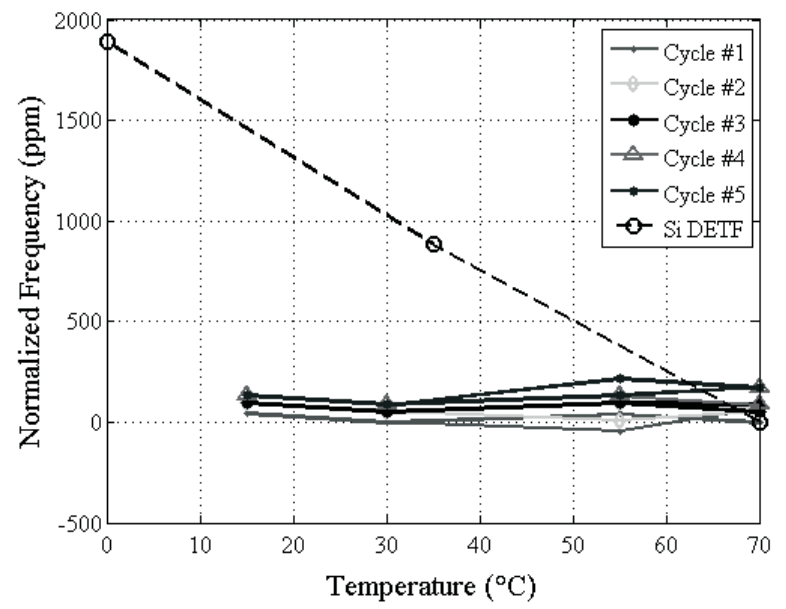

Figure 4. Frequency vs. temperature variation of an oxidized DETF over five cycles from $15^{\circ} \mathrm{C}$ to $70^{\circ} \mathrm{C}$. The total frequency excursion, over a $15 \mathrm{hr}$ span, was $260 \mathrm{ppm}$. However, approximately $150 \mathrm{ppm}$ could be attributed to a $\sim 10 \mathrm{ppm} / \mathrm{hr}$ drift.

\section{CONCLUSION}

Preliminary results show that it is possible to passively reduce the TCF of silicon resonators by incorporating Silicon Dioxide into the structure of the resonator. A relation for the TCF of a composite beam is developed and it is shown that the optimal composite configuration is achieved by oxidation of the surfaces of the silicon resonator. Devices were fabricated in a wafer-scale oxide seal processes and show a 10x reduction in the native TCF compared to single material Silicon resonators. In future work, a combination of passive and active compensation will be used to achieve a TCF less than 1 ppm over this same range.

\section{ACKNOWLEDGEMENTS}

This work was supported by DARPA HERMIT (ONR N66001-018942), The National Nanofabrication Users Network facilities funded by NSF (ECS-9731294), The National Science Foundation Instrumentation for Materials Research Program (DMR 9504099), and the Stanford Graduate Fellow support for R. Melamud. The authors would like to thank Gary Yama and Eric Peroziello for their invaluable help and guidance.

\section{REFERENCES}

[1] W.-T. Park, et al. Technical Digest of Transducers (2003)

[2] M.A. Hopcroft, et.al. Technical Digest Hilton Head (2004)

[3] W-T. Hsu, et.al. Technical Digest of MEMS (2002)

[4] W-T. Hsu, et.al. Technical Digest IEDM. (2000)

[5] Melamud, et.al. Technical Digest Transducers (2005)

[6] McSkimmin, Journal of Applied Physics. 24 (8), (1953)

[7] Plummer, et.al. "Silicon VLSI Tecnology", (2000) 